\title{
La ecología política de las asociaciones empresariales españolas en el sector de la información y las comunicaciones*
}

Achim Lang

\begin{abstract}
Resumen: Este artículo analiza los procesos de reforma y adaptación de las asociaciones empresariales españolas provocados por los cambios en el entorno político y económico. Las innovaciones tecnológicas, junto con un fuerte ritmo de crecimiento muy elevado, ha dado lugar al surgimiento de nuevos tipos de asociaciones empresariales en el sector de la información y comunicación. La incorporación de estas nuevas asociaciones en las estructuras de representación de intereses tradicionales se caracteriza, principalmente, por la escasa inclusión en las asociaciones nacionales más importantes. No obstante, en el caso de España, la CEOE ha adoptado una posición que favorece esta inclusión, facilitando así la consolidación de los mecanismos de representación de intereses más recientes.
\end{abstract}

Palabras clave: Grupos de interés, asociaciones empresariales, redes sociales, cooperación, competición, ecología política.

\section{INTRODUCCIÓN}

La transferencia de teorías de la biología a la ciencia política es un fenómeno relativamente nuevo. Desde los años noventa, sin embargo, ha aumentado la cantidad de publicaciones que analizan planteamientos en la ciencia política por medio de teorías de la evolución. Junto a introducciones y reflexiones generales sobre la biología y el darwinismo en la ciencia política (Masters, 1990; Anhart, 1994; Kerr, 2002) existen una serie de estudios que hacen referencia a estas teorías para aclarar no solo cambios en las instituciones (Jones et al., 2003; Ward, 2003) y el surgimiento de políticas públicas (John, 1999; Steinmo, 2003), si no también la interacción entre diferentes actores políticos (Ronit y Schneider, 1996). Desde hace algunos años se han empeza-

* El autor agradece a Laura Albarracín y Karina Frainer su impagable apoyo lingüístico. 
do a utilizar ideas provenientes de las teorías de la evolución o de la ecología en las investigaciones sobre las asociaciones empresariales, generando una nueva alternativa teórica neo-pluralista frente a la perspectiva neo-corporativista (McFarland, 2004; Lowery y Gray, 2004). Estas nuevas teorías hacen especial referencia, por un lado, al paralelismo entre los procesos de diferenciación económica y social y, por otro lado, a su impacto en el panorama de las asociaciones empresariales. Frente a estas teorías, aquellos que defienden el desarrollo de teorías desde una perspectiva neo-corporativista, perciben la organización jerárquica como un factor vital para la reducción de la complejidad en la configuración de las asociaciones empresariales.

Este artículo retoma esta controversia para analizar las relaciones entre asociaciones empresariales. En este contexto se analiza la diferenciación y el surgimiento de relaciones ecológicas y de coordinación en las asociaciones empresariales españolas en un sector que se estableció hace relativamente poco tiempo: el sector de la información y las comunicaciones. El enfoque de este artículo plantea como pregunta hasta qué punto el panorama de las asociaciones empresariales en este sector presenta una estructura de redes similares al panorama nacional de las asociaciones empresariales. En el periodo posterior a la transición democrática, la estructura de representación nacional de los empresarios tomó la forma de una organización jerárquica centrada alrededor de la Confederación Española de Organizaciones Empresariales (CEOE), en la cual confluyen todas las asociaciones de los diferentes sectores económicos. La CEOE representa alrededor de un millón de empresas que están organizadas en cerca de dos mil asociaciones regionales y doscientas sectoriales. Las asociaciones empresariales acopladas a esta estructura tienen sin embargo una amplia autonomía. De hecho, la estructura de las asociaciones empresariales españolas se caracteriza por su fuerte segmentación y por las relaciones formales e informales de cooperación, en su mayoría con las asociaciones centrales de los diferentes sectores (Pardo y Fernández, 1991; Molins y Morata, 1993; Molins y Casademunt, 1999). De acuerdo a este panorama, las asociaciones empresariales españolas del sector de la información y las comunicaciones habrían debido desarrollar redes de cooperación en las cuales las asociaciones pertenecientes a la CEOE tuvieran un rol central. Estas redes de cooperación deberían caracterizarse por su fuerte centralización y por el predominio de una asociación central sectorial.

Los datos usados en este artículo se basan en entrevistas estandarizadas hechas a representantes (en su mayoría gerentes) de alrededor de 14 asociaciones empresariales ${ }^{1}$. Estas consultas fueron llevadas a cabo entre septiembre de 2002 y julio de 2003, y fue analizado el periodo 1987-2002. El año 1987 fue elegido como inicio del periodo de análisis, ya que en este año se publicó el libro verde de la Comisión Europea, que fue determinante para el inicio de una política común de telecomunicaciones europea.

\footnotetext{
4 ca de 150 representantes de asociaciones empresariales en cinco países. Más información acerca de este tema se encuentra en Grote et al. (2008) y Lang (2006).
} 
El artículo está dividido, además de la introducción, en tres secciones. La segunda sección se enfoca en la clasificación de teorías ecológicas en la investigación de las asociaciones empresariales. La tercera sección describe el diseño de la investigación y hace una operacionalización de las relaciones entre las asociaciones empresariales. Finalmente se analiza la ecología de las redes formadas por las asociaciones empresariales, al igual que las relaciones informales de cooperación que predominan en éstas, describiéndose también su surgimiento.

\section{LAS REDES SOCIALES EN LA INVESTIGACIÓN DE ASOCIACIONES EMPRESARIALES: LA ECOLOGÍA DE LAS ASOCIACIONES EMPRESARIALES}

La ecología, como disciplina de la biología, es la ciencia de la distribución y la frecuencia de organismos, así como también de las interacciones que determinan esta distribución y frecuencia. De esta manera, las interacciones o relaciones ecológicas son centrales para el análisis de poblaciones biológicas que comparten un mismo hábitat, y para el caso de las asociaciones empresariales, de poblaciones organizacionales que comparten un mismo sector político. En un hábitat, las poblaciones biológicas compiten por alimentos y lugares de incubación. Sin embargo, también se construyen vínculos de cooperación entre las poblaciones, que contribuyen a su supervivencia diaria. En la investigación de poblaciones en ecología se analizan las relaciones entre poblaciones de manera sistemática y se clasifican a largo de la dimensión competencia-reciprocidad. De acuerdo con los esquemas de clasificación en ecología, existe, por ejemplo, una relación simbiótica entre dos poblaciones, cuando las dos poblaciones se benefician de la relación (Townsend et al., 1996).

En la ciencia que investiga las organizaciones, esta clasificación de las relaciones ecológicas ya fue introducida hace algún tiempo. Brittain y Wholey (1998) diferencian seis tipos de interacciones entre las poblaciones de organizaciones de la industria de semiconductores estadounidense, y analizan los efectos de las estructuras relacionales en el desempeño de estas:

- Interacción inexistente: entre la pareja de asociaciones empresariales no existe ningún tipo de relación, ni de cooperación o de competencia.

- Neutralidad: entre ambas asociaciones no existe ninguna relación, o existen ambas relaciones (cooperación y competencia) simultáneamente.

- Comensalismo: una de las asociaciones no mantiene ninguna relación, o ambas relaciones simultáneamente, mientras que la otra asociación coopera.

- Mutualismo: ambas asociaciones cooperan y no tienen relaciones de competencia.

- Competencia parcial: una de las asociaciones no tiene ninguna relación, o ambas relaciones simultáneamente, mientras que la otra asociación mantiene una relación de competencia. 
- Competencia total: ambas asociaciones compiten y no tienen relaciones de cooperación.

- Competencia “perjudicial”: mientras que una asociación coopera, la otra compite.

La diferenciación de relaciones ecológicas entre poblaciones es hasta ahora un terreno desconocido para la investigación de las asociaciones empresariales. Especialmente la investigación neocorporativista se ha concentrado hasta ahora en la descripción y el análisis de jerarquías formales e informales. En este marco Schmitter y Streeck subsumen, dentro del término "estructuras inter-organizacionales", a las "higher-order associations" por un lado y a los sistemas de asociaciones empresariales por otro. Las asociaciones de alto rango son definidas de la siguiente como organizaciones "con un staff y una constitución cuyos miembros son otros BIAs. En términos prácticos, las higher-order associations son organizaciones permanentes especializadas en la coordinación de las actividades de los miembros a ella asociados" (Schmitter y Streeck, 1999: 69). Contrario a esto, los sistemas de asociaciones empresariales cobijan a todas las asociaciones activas (incluidas las asociaciones de alto rango) en un campo determinado. La diferenciación e integración dentro de estos sistemas de asociaciones empresariales puede llevarse a cabo a lo largo de una dimensión horizontal o vertical (Schmitter y Streeck 1999: 72-78).

La diferenciación horizontal surge cuando dentro de un sector se distribuyen los diferentes campos de acción entre las distintas asociaciones empresariales, permitiendo a estas especializarse en una parte específica. En aquellos sistemas de asociaciones empresariales no regulados, pueden generarse relaciones de competencia cuando más de una asociación se especializa en el mismo campo. Por otro lado, la diferenciación vertical surge con la existencia de asociaciones de alto rango. Estas coordinan las actividades de las asociaciones en los niveles inferiores y de esta manera contribuyen a la creación de una integración jerárquica dentro del sistema de asociaciones (Coleman y Grant, 1988; Coleman y Montpetit, 2000). Por el contrario, la integración horizontal se caracteriza por la construcción de mecanismos de coordinación no jerárquicos entre las asociaciones. La construcción de alianzas formales e informales es un ejemplo de estos mecanismos de coordinación.

A partir de este análisis sobre las distintas formas de integración horizontal y vertical, Schmitter y Streek (1999) distinguen dos tipos ideales de sistemas de asociaciones empresariales. Los sistemas de asociaciones empresariales corporativistas se caracterizan por las relaciones estables y duraderas en todos los niveles del sistema. En este panorama dominan las relaciones de cooperación y coordinación entre los diferentes niveles jerárquicos del sistema. Esta estructura jerárquica culmina con la asociación de alto rango, que es incluida en el proceso de formulación de políticas públicas. Esta lógica se caracteriza por procesos de intercambio entre la asociación de alto rango y las entidades estatales que les permite a ambos actores adquirir re46 cursos, los cuales serían imposibles de obtener sin este intercambio. Las relaciones 
de cooperación también son predominantes en este marco. Esto es igualmente válido para los sistemas de acción colectiva con una lógica de afiliación (logic of membership). Las empresas miembro se afilian a estos sistemas a causa de los incentivos ofrecidos por las asociaciones regionales o las asociaciones profesionales.

Los sistemas de asociaciones empresariales pluralistas son, al contrario, fundamentalmente más fluidos y dinámicos. En relación a la articulación de intereses entre las asociaciones y el estado no se establecen por ejemplo, estructuras estables de jerarquía, dado que el dominio de una sola asociación genera por lo general una reacción contraria por parte de los intereses no representados (Truman, 1951). De manera similar, la relación entre las asociaciones y sus miembros es inestable, ya que ninguna asociación puede garantizar una afiliación constante. La competencia es la lógica dominante en todos los niveles, especialmente acentuada en aquellos sistemas que se encuentran en una constante "batalla" por obtener miembros y acceso a aquellos actores determinantes en los procesos políticos.

El interés principal de este artículo son las relaciones internas de los sistemas de asociaciones empresariales, así como la interacción entre las asociaciones y los distintos actores estatales. En la siguiente sección se explicara la selección de asociaciones empresariales sobre las que se basa nuestro análisis. Además, se aclarará la identificación de relaciones ecológicas de acuerdo con los métodos cuantitativos de análisis de redes sociales.

\section{RECOLECCIÓN DE DATOS Y METODOLOGÍA}

La selección de los actores analizados en el estudio de las redes sociales es de vital importancia para la validez de los resultados de dicho análisis. En general, se puede diferenciar entre tres estrategias para la selección de dichos actores. Por un lado, se pueden analizar todos los actores pertenecientes a una red. Sin embargo, por motivos pragmáticos en la investigación, esta forma de selección no suele adoptarse. De manera similar, tampoco se aconseja la toma de muestras, dado que en la mayoría de los casos en los que se analizan redes sociales, el interés se encuentra en examinar las relaciones entre los actores más relevantes y no en la recopilación de datos promedio. La tercera estrategia es entonces llevar a cabo un análisis que se limita a los actores mas importantes y centrales en la red. Para seleccionar estos actores centrales, se debe, sin embargo, llevar a cabo una preselección de todos los actores que pertenecen a una red. En el caso del análisis de las asociaciones empresariales españolas en el sector de información y comunicaciones, esta preselección se llevo a cabo por medio de listas e investigación en Internet. Además, se utilizó un muestreo tipo "bola de nieve", por medio del cual se analizaron las páginas Web de todas las asociaciones empresariales del sector, para así establecer posibles conexiones con otras asociaciones. Luego de esta intensiva investigación, pudimos preseleccionar un total de cincuenta y tres asociaciones en el sector de la información y las comunicaciones español. 
La selección de asociaciones focales en este sector se llevó a cabo por medio de otros criterios. Laumann (1983) diferencia entre dos métodos de selección: el nominal y el real. En el proceso de selección por medio del método nominal, los actores son seleccionados a causa de sus características específicas, o del grupo al que pertenecen, como pueden ser por ejemplo la antigüedad de la asociación o su pertenencia a una asociación de alto rango. Sin embargo, dada la inexistencia de criterios significantes que permitan una clasificación nominal, se utilizará en este análisis el método real. La selección se llevó a cabo mediante este método, mediante encuestas a expertos llevadas a cabo en tres países. Como expertos se entiende a investigadores con experiencia en este sector, y también a funcionarios públicos conocedores del sector. A estos se les pidió evaluaran la relevancia de las asociaciones en el proceso político en una escala de cero a dos, siendo "cero = insignificante", "uno = importante" y “dos = muy importante". De la suma de opiniones de los expertos resultó un rango de reputación de las asociaciones en cada país. Para este análisis fueron seleccionadas aquellas asociaciones que recibieron más de $25 \%$ del puntaje máximo establecido en el rango de reputación hecho por lo expertos. Este proceso llevó a la selección de un total de 18 asociaciones empresariales de las 53 que fueron preseleccionadas en España. De estas 18, finalmente 14 participaron en la investigación.

En la investigación de asociaciones empresariales se identifican, en su mayoría, dos tipos de relaciones. Por un lado, la relación caracterizada por la competencia en los sistemas pluralistas, y por otro lado la relación determinada por la cooperación y la subordinación en los sistemas jerárquicos y corporativistas. De esta manera, buscamos hasta que punto predominan en los sistemas de asociaciones empresariales las relaciones de competencia o cooperación. La red ecológica de relaciones que será analizada en este artículo expone las diferentes relaciones de cooperación, neutralidad y competencia entre las asociaciones. Para poder analizar esta red social se identifican dos formas diferentes de relación entre las asociaciones. Los representantes de las asociaciones debieron indicar con que otras asociaciones mantienen una relación o una relación muy importante, y con cuales existe una relación de competencia, ya que ambas asociaciones se especializan en el mismo campo. Finalmente se llevo a cabo el cálculo de esta estructura de relaciones por medio del algebra de matrices.

El cálculo de la red de cooperación se basa en preguntas hechas a las asociaciones respecto al intercambio de información entre ellas. Con este fin, se les preguntó a los representantes de las asociaciones a que organizaciones enviaban información y de cuales la recibían. Para determinar la jerarquía y la dominancia de una asociación en una red social se calcula el grado de centralidad y la centralidad intermedia (Wassermann y Faust, 1994: 169-219). El cálculo de estos dos valores garantiza que todos los posibles aspectos de la posición de la asociación en la red social sean incluidos en el análisis.

El grado de centralidad es una medida estadística para la centralidad de un actor, la cual se basa única y exclusivamente en las relaciones directas que mantiene este 48 actor con otros actores dentro de una red social. Según esta medida, se define como 
un actor central a aquellos actores con muchos "vecinos" en la red. Es decir, aquellos actores cuya posición colinda con la de muchos otros dentro la red social. En redes sociales en las cuales las relaciones son dirigidas se puede diferenciar entre dos grados de centralidad: el grado de centralidad de entrada (indegree) y el de salida (outdegree). El máximo valor se logra cuando un actor mantiene con todos los demás actores una relación de entrada o de salida.

La centralidad intermedia es una medida de distancia, por medio de la cual se calcula la centralidad de un actor en una red social, identificando la distancia física entre este actor y los otros. La idea es que un actor es central siempre y cuando esté situado en el camino más corto para llegar a los otros actores (Wassermann y Faust, 1994: 188-192). Si existen varios caminos cortos, se asume que todos tienen la misma probabilidad de ser usados. La centralidad intermedia es especialmente apropiada para identificar jerarquías informales en las redes sociales. Por ejemplo, en el caso de una red social que toma la forma de una estrella, el actor que se encuentra en el centro de esta, tiene un valor de centralidad intermedia de uno, dado que en este caso es imposible para los otros actores tener caminos más cortos de comunicación. En una red social completa en la cual todos los actores están conectados los unos con otros, todos tienen una centralidad intermedia de $1 / n$.

\section{CONFIGURACIÓN, COORDINACIÓN Y LOBBY DE LAS ASOCIACIONES EMPRESARIALES}

La población de asociaciones empresariales españolas experimentó un fuerte crecimiento en los últimos veinte años. A mediados de los años ochenta existían tan solo ocho asociaciones empresariales en los sectores de las comunicaciones, los bienes electrónicos, los medios impresos y la industria de la publicidad (pertenecientes a nuestro grupo de asociaciones centrales). Entre las asociaciones del sector de las comunicaciones y los bienes electrónicos se encuentra la Asociación Nacional de Industrias Electrónicas y Telecomunicaciones (ANIEL) y la Asociación Española de Empresas de Tecnologías de la Información (SEDISI). ANIEL fue fundada en 1973 como la asociación encargada de representar la industria electrónica, y hoy en día representa además a la industria de telecomunicaciones (ANIEL, 2002). En este marco, representan los intereses de los productores de componentes electrónicos, al igual que los de las empresas de telefonía españolas y las compañías asociadas a estas. Frente a ANIEL, que representa los intereses de los productores de hardware, SEDISI organiza desde su fundación en 1976 los intereses de las empresas en el sector de las telecomunicaciones y la informática (SEDISI, 2000). En 1984 por medio de la fusión con la asociación empresarial de software ASOFT, SEDISI logró un monopolio en la representación del sector de la informática.

El grupo de los medios impresos e industria de la publicidad fue fundado antes de 1987 y comprende seis asociaciones empresariales. La Asociación de Editores de Diarios Españoles (AEDE) pertenece al sector de los medios impresos y fue fundada 
en 1978, representando los intereses de los editores de diarios españoles. De acuerdo con informaciones de AEDE, tiene un grado de organización del 90\% y en el año 2000 también se convirtió en la asociación patronal que representa los intereses de los empresarios en el ámbito de la negociación colectiva. Esta transformación se consolidó por medio de un cambio en los estatutos de AEDE aprobado por la asamblea general de la misma².

Los editores de revistas se organizan a su vez en dos asociaciones principales. La Asociación de Revistas de Información (ARI) representa los intereses de los editores de revistas de interés común, que en 1977 se separaron de la Agrupación Nacional Sindical de Revistas, para así fundar ARI. Pertenecen a ARI 37 grupos editoriales, lo cual comprende más de 400 revistas, es decir cerca de $80 \%$ del mercado nacional español. Por otro lado, la Asociación de Prensa Profesional (APP) representa a las revistas técnicas. Esta última fue fundada en 1925 bajo el nombre de Asociación Española de Prensa Técnica (AEPT) con el fin de representar los intereses de la prensa técnica. In 1933 cambió su nombre a APP, para así no representar solo a la prensa técnica sino también a la prensa profesional en general.

\section{FIGURA 1}

El desarrollo de la población de asociaciones empresariales a partir de 1987

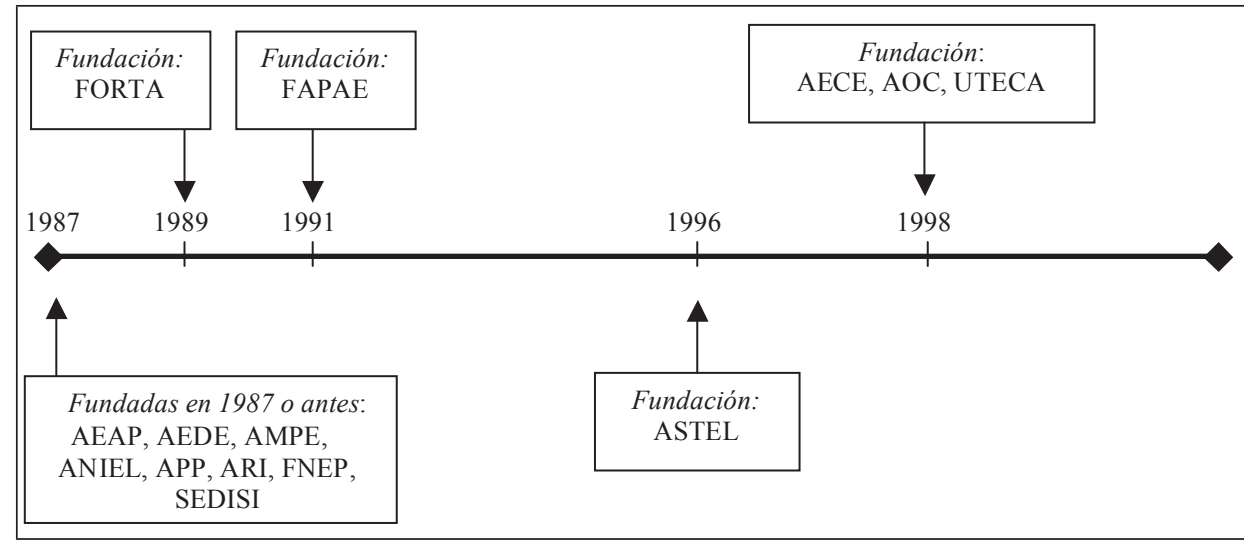

Fuente: Elaboración propia.

La industria de la publicidad está representada por tres principales asociaciones ô empresariales: la Federación Nacional de Empresas de Publicidad (FNEP), la Asociación Española de Agencias de Publicidad (AEAP), y por último, la Asociación de Medios Publicitarios de España (AMPE). FNEP, fundada en 1978, es una asociación de alto rango que no solo representa a las doce asociaciones publicitarias regionales,

2 Fuente: El País, 27 de octubre de 1999. 
sino también actúa como asociación patronal en este sector. Por otro lado, AEAP que fue fundada en 1977, es la asociación encargada de representar los intereses de las agencias de publicidad. Estas ultimas se encargan no sólo de crear los contenidos publicitarios, sino también de la publicación de estos en los diferentes medios de comunicación. Por último, AMPE creada en 1963, es la asociación encargada de representar los intereses de aquellas empresas que transmiten la publicidad a través de diferentes medios de comunicación. De esta manera, AEPE conecta los intereses de los productores de publicidad con los de las empresas encargadas de transmitirla, como lo son por ejemplo las empresas de radiodifusión o la prensa.

A partir del año 1987 se integraron seis nuevas asociaciones empresariales al grupo de asociaciones focales español (Figura 1). Ya para el año 1989 fue creada la Federación de Organismos de Radio y Televisión Autonómicos (FORTA). Sin embargo, FORTA no tiene asociaciones miembro, sino que representa a ocho operadores regionales de radiodifusión (en 2002). La fundación de FORTA puede ser vista como reacción al cambio en el panorama español de la radiodifusión a partir de los años ochenta. A raíz del surgimiento de estaciones radiales y televisivas privadas, tanto nacionales como locales, los actuales miembros de FORTA se vieron expuestos a una alta competencia, que decidieron combatir por medio de una intensa cooperación, que fue más allá de una simple cooperación regional. De esta manera, FORTA no solo cumple las tradicionales funciones de lobby, sino que también se encarga de la adquisición de derechos de transmisión cinematográficos y de eventos deportivos. En este campo, FORTA lleva a cabo, en representación de sus miembros, las negociaciones con los productores de Hollywood y con la confederación de fútbol española. Similarmente, en el campo de la radiodifusión se estableció en 1991 la Federación de Asociaciones de Productores Audiovisuales Españoles (FAPAE). Esta representa los intereses de los productores de televisión y cine en España. FAPAE esta compuesta, por un lado, por las asociaciones de las regiones autónomas, y por otro por las asociaciones pertenecientes a las diferentes ramas en el sector, como por ejemplo la Asociación de Productoras Independientes (API) o la Asociación de Productores de Publicidad de España (APPE). Con alrededor de 350 miembros, FAPAE representa a todos los productores audiovisuales en España (FAPAE 2002). Como última asociación focal, se fundó en 1998 la Unión de Televisiones Comerciales en Abierto (UTECA), con el fin de proteger los intereses de los programas televisivos comerciales. UTECA tiene tan solo tres miembros (en 2002). Estos, sin embargo, pertenecen a las cadenas de televisión españolas más grandes.

Las otras tres asociaciones fundadas a partir de 1987 pertenecen al sector de las telecomunicaciones. La Asociación de Empresas Operadoras y de Servicios de Telecomunicaciones (ASTEL) fue fundada en 1995 y representa a las empresas de telecomunicación españolas, con excepción de Telefónica. ASTEL se perfila como el polo político que se opone al monopolio de la Telefónica y al fuerte contacto de esta última con las entidades estatales españolas (ASTEL 2000). La Agrupación de Operadores de Cable (AOC) fundada en 1998 une los intereses económicos y políticos de los operadores de cable regionales. De manera similar a la FORTA, AOC no solo representa los 
intereses políticos de sus miembros, sino que también interviene en la creación de una política económica común. En el interior de la AOC, por ejemplo, se coordinan y aprueban los planes de inversión para la ampliación de las redes y la infraestructura en el sector³. También en el marco de una "joint venture" de los operadores de cable, AOC cierra acuerdos para la adquisición de derechos de transmisión de eventos deportivos $^{4}$. En el mismo año que fue fundada AOC también se creo la Asociación Española de Comercio Electrónico (AECE), creada bajo el incentivo de la Asociación Española de Marketing Directo (AEMD), que tiene treinta grandes empresas afiliadas 5 . A su vez, la AEMD se desarrolló de manera paralela en una federación hoy en día conocida como la Federación de Comercio Electrónico y Marketing Directo (FECEMD). Esta federación agrupa siete asociaciones profesionales, y de esta manera cubre todas las ramas en el sector del comercio electrónico y el marketing directo. AECE es sin lugar a duda la asociación miembro más importante de IFECEMD. Tan solo en el año 1999, AECE organizó el trabajo de la mitad de las asociaciones pertenecientes a la federación (AECE, 2001; FECEMD, 2001). La gerente de AECE es a su vez gerente de FEDEMD. La importancia de esta asociación de comercio electrónico para FEDEMD fue reconocida en el año 2003, cuando esta última recibió el nombre de AECE-FECEMD.

En resumen, se puede establecer que la configuración de la población de asociaciones empresariales en el sector de información y comunicaciones se transformó de manera significativa a partir del año 1987. Casi la mitad de las asociaciones fueron fundadas después de este año, tanto en el sector de las telecomunicaciones como en el de la radiodifusión. Sin embargo, vale la pena recalcar que al margen de la creación de las nuevas asociaciones, no se evidenciaron otros cambios en la configuración de la población de asociaciones empresariales. Transformaciones tales como separaciones, adquisiciones o fusiones no se llevaron a cabo. La fundación de nuevas asociaciones en el sector de información y comunicaciones también trajo consigo cambios en el panorama nacional de asociaciones empresariales. La CEOE, como organización cúpula, supo, no obstante, reaccionar de manera adecuada a la nueva estructura de poder en este sector e integró a las nuevas asociaciones de telecomunicaciones e Internet. Un total de siete asociaciones empresariales focales pertenecen a la CEOE (en 2002). Entre estas se encuentra la AECE (por medio de la FECEMD), ANIEL, AOC, ASTEL, FAPAE, FNEP y SEDISI. De esta manera, la mayor parte del sector se encuentra representado en la CEOE.

\subsection{Relaciones ecológicas entre las asociaciones empresariales}

En la Figura 2 se puede observar una representación de la estructura de las relaciones entre las asociaciones empresariales sectoriales. Dentro de este sistema no están integradas la AECE y la AOC, que fueron fundadas recientemente. Las demás asociaciones se agrupan en dos clusters cohesivos. 
En el primer cluster se encuentran seis asociaciones empresariales pertenecientes a los sectores de los medios de comunicación y la industria de la publicidad. Entre estas asociaciones existen tanto relaciones de cooperación y competencia, como también relaciones neutrales.

\section{FIGURA 2}

La red de las relaciones de las asociaciones empresariales

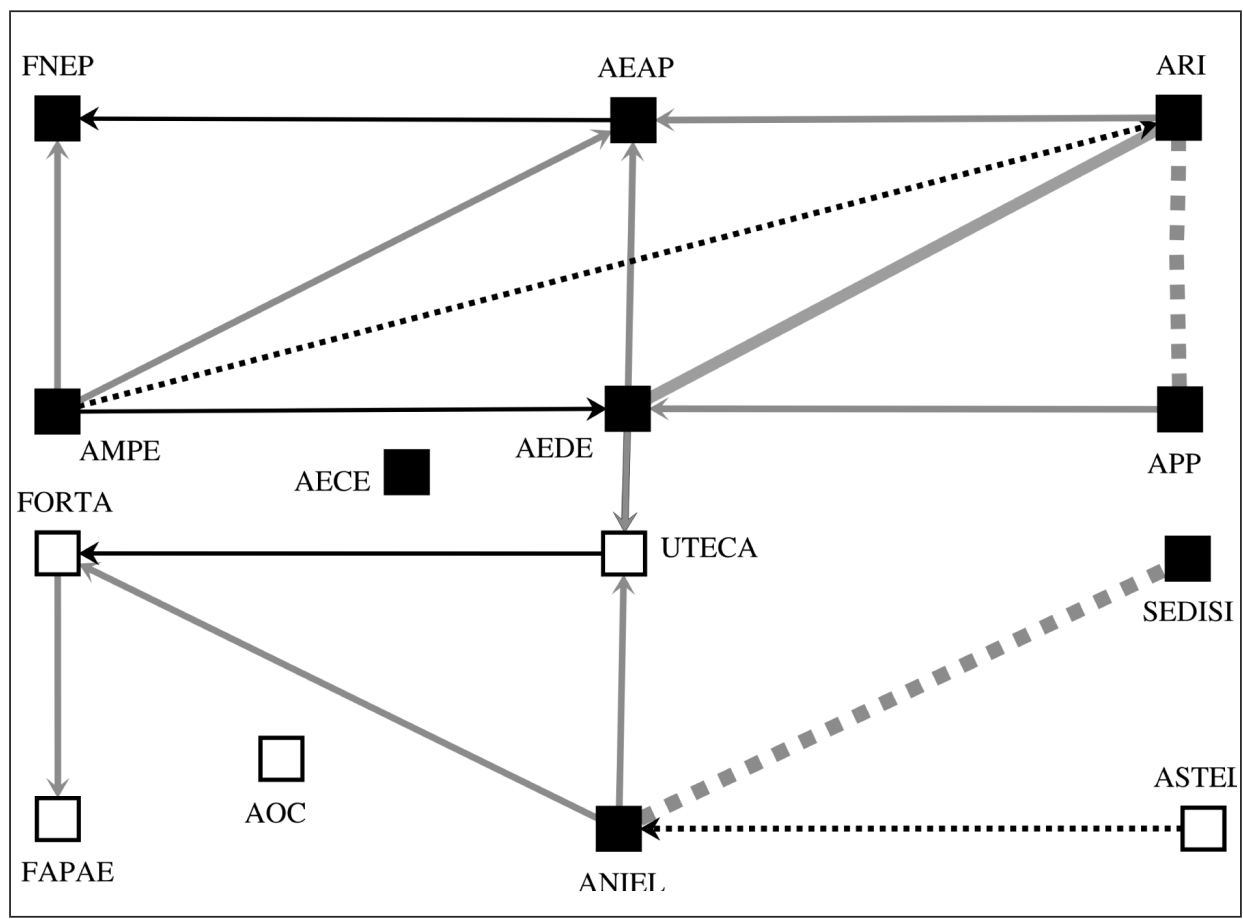

Fuente: Elaboración propia.

Relaciones: Línea ancha negra (sin flecha): Competencia total. Línea delgada negra (con flecha): Competencia parcial, flecha en dirección al competidor. Línea punteada negra (con flecha): Competencia perjudicadora, flecha en dirección al perjudicado.

Línea ancha gris (sin flecha): Mutualismo.

Línea delgada gris (con flecha): Comensalismo, flecha en dirección al beneficiado. Línea punteada gris: Neutralidad.

Asociación: Negro: asociación establecida.

Blanco: asociación fundada a partir de 1987 o fruto de la fusión con una asociación anterior.

Las relaciones de competencia existen única y exclusivamente entre las asociaciones empresariales pertenecientes a la industria de la publicidad. AMPE se encuentra en directa competencia con AEDE y ARI, ya que estas últimas asumen la representación de 
sus miembros en temas relacionados a la publicidad6 (El País 9.03.2002: 28). Las asociaciones de prensa o revistas se comportan, contrario al panorama anterior, de manera cooperativa o neutral. ARI y APP mantienen relaciones neutrales. Ambas asociaciones limitan su actividad a la representación de intereses en sus respectivos campos, es decir al campo de la prensa profesional por un lado, y al de la prensa técnica por el otro. Una cooperación formalizada, o una distribución de competencias como tal, no existen.

Encontramos una relación de cooperación que abarca más de un sector entre las asociaciones de diarios y revistas españolas AEDE y ARI, y la asociación de televisión comercial UTECA. Estas asociaciones actúan conjuntamente contra la institución pública de radio y televisión española RTVE, que con su agresiva política publicitaria genera perdidas para los otros medios de comunicación?. Ya para el año de 1997, la AEDE había iniciado una campaña contra los procedimientos de la RTVE, que sin embargo, no generó ningún efecto ${ }^{8}$. Como medida para fortalecer su campaña AEDE buscó aliados, que encontró en la entonces recientemente fundada UTECA ${ }^{9}$ y en $\mathrm{ARI}^{10}$. Esta confrontación se agudizó aun más, dada la intervención del Ministro de Finanzas a favor de la RTVE"1․

En el segundo cluster en la Figura 2 se encuentran asociaciones empresariales pertenecientes a los sectores de las telecomunicaciones y la radiodifusión. La densidad de la relaciones en este cluster es claramente menor a las del cluster anterior. Este cluster está compuesto por sólo dos asociaciones empresariales establecidas: ANIEL y SEDISI. Entre ambas asociaciones existe una relación neutral, caracterizada por una clara distribución de competencias. Solamente en aquellos casos, en los cuales los intereses comunes se ven afectados, existe una cooperación entre ambas asociaciones. De esta manera, por ejemplo, ambas asociaciones iniciaron, en cooperación con el Ministerio de Ciencia y Tecnología, la campaña informativa "Info XXI". Esta campaña tenía el fin de cultivar una nueva generación de personas calificadas para trabajar en el sector de la electrónica e informática, al igual que una readaptación profesional en los oficios relacionados a la tecnología de la información para los desempleados ${ }^{12}$. Otro ejemplo de cooperación fue la campaña iniciada por ANIEL y SEDISI, y a la cual se adhirió ASTEL, en contra de la decisión del Ministerio de Fomento de aumentar las tarifas para las llamadas locales e internacionales ${ }^{13}$.

La Asociación Nacional de Industrias Electrónicas y Telecomunicaciones (ANIEL) coopera a su vez con las asociaciones de radiodifusión FORTA y UTECA, las cuales mantienen una relación de competencia. En el año 1998 las tres asociaciones colabo-

6 Fuente: El País, 9 de marzo de 2002.

7 Fuente: El País, 9 de marzo de 2002.

8 Fuente: El País, 22 de enero de 1997.

9 Fuente: El País, 6 de junio de 2001.

10 Fuente: El País, 9 de marzo de 2002.

11 Fuente: El País, 9 de marzo de 2002.

12 Fuente: El País, 7 de octubre de 2001.

13 Fuente: El País, 2 de julio de 1998. 
raron con un proyecto del Ministerio de Fomento que buscaba no solo aumentar las frecuencias terrestres, sino también conferir nuevas licencias de radiodifusión. ANIEL esperaba que un aumento en la competencia en el sector de la radiodifusión se viera reflejado en un aumento en el mercado de televisores y otros equipos de radiodifusión. Entre FORTA y UTECA por el contrario, se dieron discrepancias respecto a la cantidad de frecuencias y licencias que pudieran ser adquiridas por un solo proveedor. UTECA criticó particularmente la posibilidad de trasmitir programas regionales a nivel nacional, los cuales serían una directa competencia para la televisión comercial, la cual representa la UTECA. Adicionalmente, las cadenas regionales integradas en FORTA tendrían privilegios a la hora de comprar programas televisivos ${ }^{14}$. Con la introducción de la televisión digital, la ANIEL y la FORTA repitieron sus exigencias. En el año 2000, ambas asociaciones se movilizaron por un acceso libre a la televisión digital, al igual que por la libre introducción de los respectivos decodificadores ${ }^{15}$.

\subsection{La coordinación de las asociaciones empresariales: la red de intercambio de información}

La integración de asociaciones empresariales en una red social con relaciones de tipo ecológico es equivalente a la cohesión estructural de una población de asociaciones empresariales. Sin embargo, basándonos en esta determinada estructura no es posible deducir si existe una cohesión funcional, que se manifieste en las actividades coordinadas de las asociaciones. Contrario a las relaciones de tipo ecológico, una red de intercambio de información puede indicar si existe una coordinación de tipo jerárquico en el sistema de asociaciones empresariales, si esta función de coordinación está distribuida entre varias asociaciones, y, finalmente, si existe una cohesión funcional dentro de la población.

La red sectorial de intercambio de información española esta compuesta, de manera similar a la red social de relaciones, por dos clusters cohesivos no ligados. La FAPAE no está integrada en ninguno de los clusters.

En el primer cluster se encuentran seis asociaciones empresariales establecidas en las industrias de medios y publicidad, al igual que la recientemente fundada asociación de televisión comercial UTECA. AEDE tiene la mayor cantidad de relaciones de información de entrada y de salida, es decir recibe y envía la mayor cantidad de información. En el cluster al cual pertenece, AEDE envía información a otras cuatro asociaciones y recibe información de cinco. Esto equivale respectivamente a un 30,8\% y $38,5 \%$ de las asociaciones empresariales focales. Por medio de su relación exclusiva con UTECA, AEDE tiene también el camino más corto para llegar a esta, y por ende goza de la mayor centralidad intermedia en este cluster. Por otro lado, AEAP y ARI constituyen, junto con AEDE y AMPE, dos grupos (cada uno con tres miembros) que

14 Fuente: El País, 3 de julio de 1998.

15 Fuente: El País, 7 de octubre de 2000. 
se sobreponen. No obstante, el intercambio de información es estos es limitado y ocasional. Solamente entre la Federación Nacional de Empresas de Publicidad (FNEP) y AMPE existe, a pesar de su carácter asimétrico, un intercambio de información constante. Finalmente, la alta densidad de este cluster se hace evidente en la poca variación de sus valores de centralidad.

FIGURA 3

La red de intercambio de información

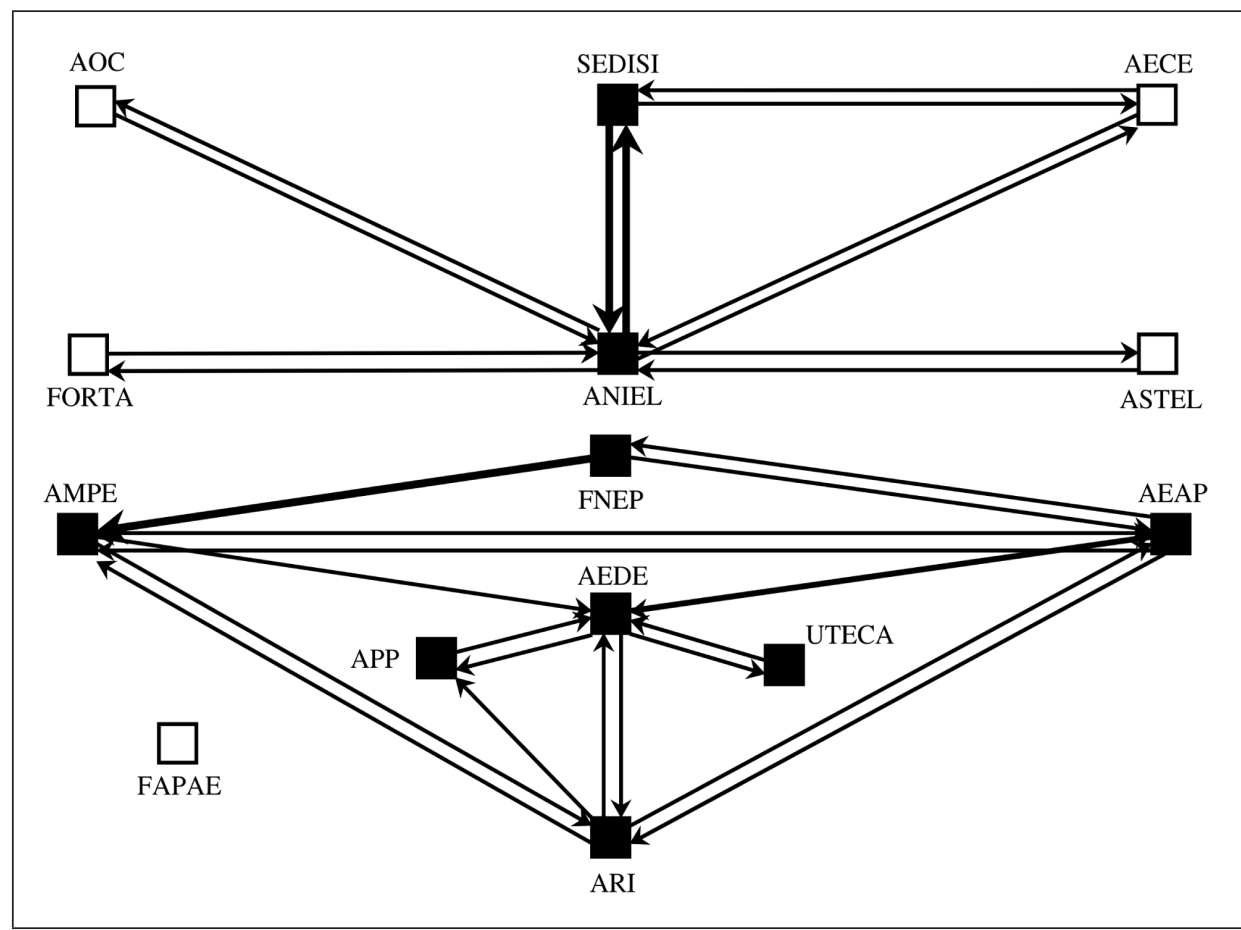

Fuente: Elaboración propia.

Relaciones: Flecha ancha: flujo de información constante.

Flecha delgada: flujo de información ocasional.

Asociación: Negro: asociación establecida.

Blanco: asociación fundada a partir de 1987 o fruto de la fusión con una asociación anterior.

Dada la alta densidad en este cluster, no existe ninguna asociación empresarial que pueda asumir un control jerárquico sobre la información. La Asociación de Editores de Diarios Españoles (AEDE) tiene sin embargo, a raíz de su relación exclusiva con UTECA, una posición destacada. Esta posición relativamente central de AEDE es confirmada por las medidas de centralidad. En este cluster se evidencian también diferencias en los valores de los distintos índices de centralidad. Los valores del grado de 56 centralidad demuestran poca variación dentro de este cluster. Esto permite concluir, 
que las asociaciones empresariales en este cluster tienen muchas relaciones directas con las otras asociaciones. El análisis de la centralidad intermedia muestra, por el contrario, que entre AEDE y las asociaciones con las cuales mantiene una relación, la distancia física es menor. AEAP, AMPE y ARI sin embargo, también disponen de "caminos cortos" para el intercambio de información. A raíz de esto, AEDE, a pesar de su posición destacada, no puede controlar el intercambio de información, ya que las otras asociaciones podrían evadir este control sus propios medios. Solamente el intercambio de información desde y hacia UTECA fluye por medio de AEDE (Figura 3).

El segundo cluster está compuesto por las asociaciones del sector de telecomunicaciones y tecnología de la información, ANIEL y SEDISI, en compañía de cuatro nuevas asociaciones pertenecientes a los sectores de las telecomunicaciones y la radiodifusión. En este cluster existe una coordinación jerárquica de las fuentes de información. Todas las asociaciones se relacionan con ANIEL e intercambian información con esta; no mantienen, sin embargo, ninguna relación entre si. Solamente la Asociación Española de Comercio Electrónico (AECE) se relaciona, aparte de con ANIEL, con SEDISI. Respecto a los índices de centralidad, este segundo cluster presenta mayor variancia que el primer cluster. En este contexto la ANIEL goza de los valores más altos e incluso es la única asociación con una centralidad intermedia mayor a cero. De esta manera, todos los caminos en la red conducen a la ANIEL. Adicionalmente, ANIEL posee una posición adyacente respecto a las demás asociaciones, lo cual le permite tener la mayor cantidad de relaciones directas con otros actores dentro de esta red social. Por consiguiente, ANIEL tiene también el mayor grado de centralidad (Tabla 1).

TABLA 1

Medidas de centralidad de las asociaciones empresariales

\begin{tabular}{|c|c|c|c|c|}
\hline \multicolumn{5}{|c|}{ Centralidad normalizada (en \%) } \\
\hline & Aislado & $\begin{array}{c}\text { Grado } \\
\text { de centralidad } \\
\text { de salida }\end{array}$ & $\begin{array}{c}\text { Grado } \\
\text { de centralidad } \\
\text { de entrada }\end{array}$ & $\begin{array}{l}\text { Centralidad } \\
\text { intermedia }\end{array}$ \\
\hline FAPAE & $\mathrm{Si}$ & 0,0 & 0,0 & 0,0 \\
\hline \multicolumn{5}{|c|}{ Cluster 1} \\
\hline AEAP & No & 30,8 & 30,8 & 5,4 \\
\hline AEDE & No & 30,8 & 38,5 & 9,9 \\
\hline AMPE & No & 23,1 & 23,1 & 2,6 \\
\hline APP & No & 7,7 & 15,4 & 0,0 \\
\hline ARI & No & 30,8 & 23,1 & 1,9 \\
\hline FNEP & No & 15,4 & 7,7 & 0,0 \\
\hline UTECA & No & 7,7 & 7,7 & 0,0 \\
\hline
\end{tabular}


TABLA 1 (cont.)

Medidas de centralidad de las asociaciones empresariales

\begin{tabular}{|c|c|c|c|c|}
\hline \multicolumn{5}{|c|}{ Centralidad normalizada (en \%) } \\
\hline & Aislado & $\begin{array}{c}\text { Grado } \\
\text { de centralidad } \\
\text { de salida }\end{array}$ & $\begin{array}{c}\text { Grado } \\
\text { de centralidad } \\
\text { de entrada }\end{array}$ & $\begin{array}{l}\text { Centralidad } \\
\text { intermedia }\end{array}$ \\
\hline \multicolumn{5}{|c|}{ Cluster 2} \\
\hline AECE & No & 15,4 & 15,4 & 0,0 \\
\hline ANIEL & No & 38,5 & 38,5 & 11,5 \\
\hline $\mathrm{AOC}$ & No & 7,7 & 7,7 & 0,0 \\
\hline ASTEL & No & 7,7 & 7,7 & 0,0 \\
\hline FORTA & No & 7,7 & 7,7 & 0,0 \\
\hline SEDISI & No & 15,4 & 15,4 & 0,0 \\
\hline
\end{tabular}

Fuente: Elaboración propia. Para el cálculo del grado de centralidad normalizado fueron codificadas las relaciones de forma binaria y llegar a una conclusión sobre el número de asociaciones "vecinas".

\subsection{Destinatarios del lobby de las asociaciones empresariales}

En la población de asociaciones empresariales españolas existe una gran concordancia entre la relevancia de una organización y la cantidad de actividades para las cuales esta organización ejerce lobby. No obstante, esta relación $(r=0,69)$ es menor en comparación a otros países como, por ejemplo Alemania o Gran Bretaña (Lang, 2006). El destinatario más nombrado del lobby de las asociaciones empresariales españolas es el Ministerio de Ciencia y Educación, el cual se encarga de la regulación de todos los sectores nacionales y del cual dependen el Consejo Asesor de Telecomunicaciones (CAT) y el Consejo Asesor de Industrias de la Sociedad de la Información (CASI).

El segundo contacto más importante de las asociaciones españolas es la asociación de alto rango CEOE. Tanto ANIEL, como AOC y SEDISI se relacionan fuertemente con la CEOE como medio para representar los intereses de sus miembros. Las demás asociaciones mantienen por el contrario, poco contacto con la CEOE. La poca frecuencia del intercambio de información con la CEOE indica que la importancia de esta es sobreestimada por parte de la mayoría de los representantes de las asociaciones.

El caso contrario es aquel de la Dirección General de Competencia de la Comisión Europea. A pesar de que esta posee la mayor relevancia entre todas las organizaciones e instituciones, solamente pocas asociaciones empresariales españolas ponen información a disposición de esta. Solamente ANIEL, ASTEL, AOC y UTECA hacen lo58 bby directo ante la Dirección General. La alta relevancia de esta organización se pue- 
de aclarar por medio de la falta de acción del gobierno español y la CMT respecto al monopolio de la Telefónica y la RTVE (Jordana et al., 2000). Este dominio se ve también reflejado en otras áreas, como por ejemplo el financiamiento de la publicidad o el suministro de servicios de Internet, y afecta de esta manera también a otras asociaciones empresariales. Estas sin embargo, no se ven afectadas en el núcleo de sus actividades, y a raíz de esto tampoco mantienen una relación directa con la Dirección General de Competencia. Por otro lado, las asociaciones empresariales españolas concentran sus actividades de lobby en las agencias nacionales, transfiriendo el papel de lobby frente a las instituciones europeas a aquellas asociaciones empresariales europeas que se desempeñan en este campo.

\section{FIGURA 4}

Los destinatarios del lobby y su relevancia

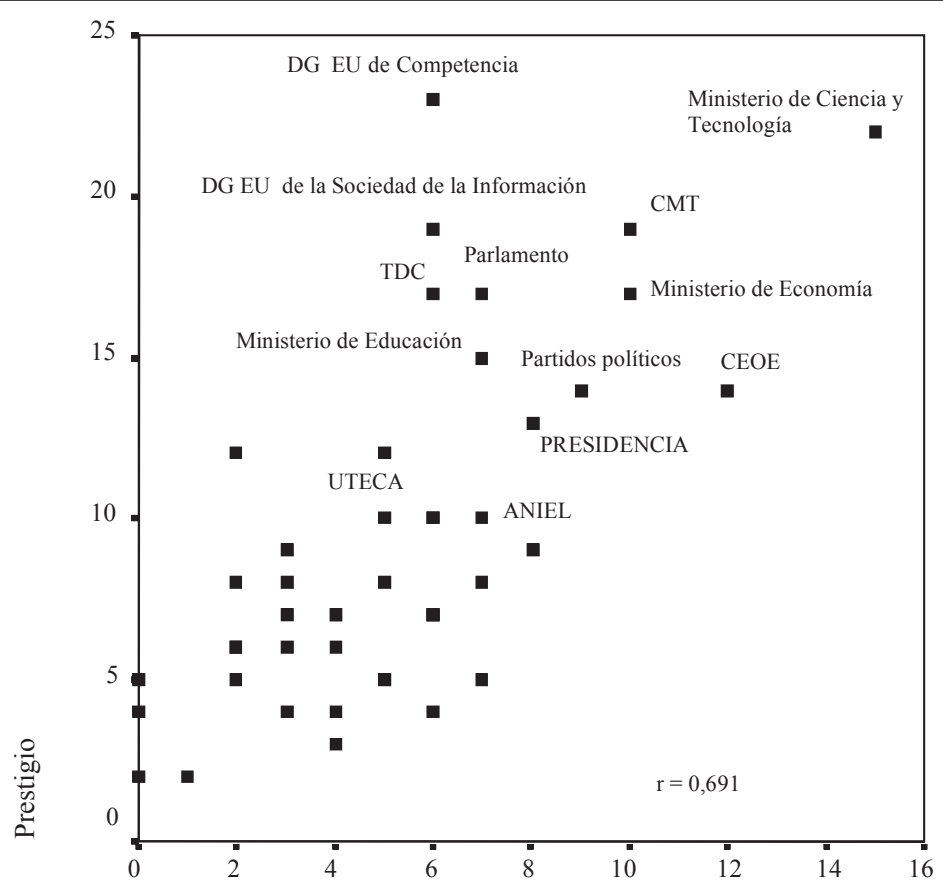

Contacto

Fuente: Elaboración propia.

Observación: La dimensión "relevancia” se calculo en base a la respuesta a la pregunta "Por favor indique las organizaciones, que desde el punto de vista de su asociación son relevantes para las actividades en su sector- 1 = relevante, 2 = especialmente relevante". La dimensión "frecuencia en el contacto" se basa en la respuesta a la pregunta "Por favor indique las organizaciones que le proveen a su asociación información importante. Por favor diferencie entre "de vez en cuando" =1, y "periódicamente” = 2". Las respuestas de las asociaciones empresariales focales fueron sumadas. 


\section{CONCLUSIÓN}

La configuración de las asociaciones empresariales españolas cambió de manera significativa entre 1987 y el 2002. Casi la mitad de las asociaciones empresariales relevantes fueron fundadas recientemente. Un total de seis asociaciones fueron fundadas, las cuales representan primordialmente los intereses del sector de la información y las comunicaciones. El crecimiento tiene su origen, por un lado, en el desarrollo económico, y por otro en la convergencia tecnológica. El surgimiento de nuevos sectores económicos y la sorprendente tasa de crecimiento de estos, llevaron a la fundación de nuevas asociaciones, las cuales escalaron rápidamente hasta localizarse en el círculo de asociaciones empresariales focales español.

Este cambio en la composición de la población de asociaciones empresariales españolas no tuvo, sin embargo, incidencia en la estructura de sus relaciones. Las nuevas asociaciones se integraron a las antiguas estructuras de relaciones sin causar fricciones de ningún tipo. No obstante, la densidad de la red social en la cual estas asociaciones están conectadas, si reduce, es como consecuencia de la integración de los nuevos miembros. En esta red social son comunes las relaciones de cooperación entre las asociaciones. Relaciones de competencia solo existen entre las asociaciones de la industria de la publicidad y la radiodifusión.

Un intercambio de información coordinado dentro de la red solo se estableció parcialmente. A excepción de FAPAE, todas las asociaciones empresariales participan en el intercambio de información. La coordinación del intercambio de información se estabilizó y el casi monopolio de ANIEL en los sectores de comunicaciones e industria electrónica se mantuvo. Las asociaciones recientemente fundadas AECE, AOC, ASTEL y FORTA se acoplaron al liderazgo de ANIEL, formando así una red social con forma de estrella y con ANIEL en el centro. Las asociaciones pertenecientes a la prensa, la industria de la publicidad y los medios de comunicación constituyen por el contrario, una red social muy densa en la cual predomina el intercambio de información reciproco entre casi todas las asociaciones. No existe una asociación central con la posibilidad de coordinar el intercambio de información. Las asociaciones empresariales se conectan en su mayoría por caminos directos, e intercambian información por medio de éstos.

Las actividades de lobby de las asociaciones se dirigen hacia los actores y las instituciones políticas más relevantes. Entre estas se encuentra la CEOE. No obstante, es interesante que esta última sea percibida por las asociaciones empresariales como un actor relevante, a pesar de que el limitado intercambio de información con esta demuestre lo contrario. En este contexto es también notorio el acceso privilegiado que tienen los miembros de la CEOE a los actores y las instituciones políticas más relevantes.

De manera concluyente se puede establecer que la influencia de la CEOE tiene 60 un efecto moderador en la estructura de las asociaciones empresariales sectoriales 
españolas. Por medio de la incorporación de las nuevas asociaciones a la estructura de la CEOE se pudo reducir la competencia entre estas, lo cual no se dio en muchos otros países (Grote et al. 2008). De esta manera, las nuevas asociaciones se pudieron integrar al panorama de asociaciones empresariales español de manera efectiva y sin mayores fricciones. Solamente en el área de los medios de comunicación, donde la influencia de la CEOE es limitada, se establecieron relaciones de competencia.

\section{REFERENCIAS BIBLIOGRÁFICAS Y DOCUMENTALES}

AECE, Asociación Española de Comercio Electrónico (2001), Memoria 1999-2000. Barcelona: AECE.

Anhart, L. (1994), “The New Darwinian Naturalism in Political Theory”, The American Political Science Review, 89: 389-400.

ANIEL, Asociación Nacional de Industrias Electronicas y de Telecomunicaciones (2002), Informe del sector electrónico y de telecomunicaciones 2001. Madrid: ANIEL.

ASTEL, Asociación de Empresas Operadoras y de Servicios de Telecomunicaciones (2000), Memoria 1999. Madrid: ASTEL.

Brittain, J.W. y Wholey, D.R. (1988), “Competition and Coexistence in Organizational Communities. Population Dynamics in Electronic Components Manufacturing”, en G.R. Carroll, ed., Ecological Models of Organizations. Cambridge, MA: Ballinger.

Coleman, W.D. y Grant, W. (1988), "The Organizational Cohesion and Political Access of Business: A Study of Comprehensive Associations", European Journal of Political Research, 16: 467-487.

Coleman, W.D. y Montpetit, E. (2000), “Multi-tiered Systems and the Organization of Business Interests", en J. Greenwood y H.J. Jacek, eds., Organized Business and the New Global Order. Basingstoke: Palgrave.

FAPAE, Federación de Asociaciones de Productores Audiovisuales Españoles (2003), Memoria Anual 2002. Madrid: FAPAE.

FECEMD, Federación de Comercio Electronico y Marketing Directo (2001), Memoria 1999-2000. Barcelona: FECEMD.

Grote, J.R., Lang, A. y Schneider, V. (eds.) (2008), Organized Business Interests in Changing Environments: The Complexity of Adaptation. Houndmills, Basingstoke: Palgrave. 
John, P. (1999), “Ideas and interests; agendas and implementation: an evolutionary explanation of policy change in British local government finance”, British Journal of Politics and International Relations, 1: 39-62.

Jones, B.D., Sulkin, T. y Larsen, H.A. (2003), “Policy Punctuations in American Political Institutions", The American Political Science Review, 97: 151-169.

Jordana, J., Ruiz, C., Sancho, D. y Sanz, A. (2000), Opiniones y Actitudes sobre las Políticas de Telecomunicaciones en España. Informe a Partir de Entrevistas a Expertos y Responsables del Sector (Noviembre 1999-Enero 2000), Barcelona: Universitat Pompeu Fabra.

Kerr, P. (2002), "Saved from extinction: evolutionary theorising, politics and the state", British Journal of Politics and International Relations, 4: 330-358.

Lang, A. (2006), Die Evolution sektoraler Wirtschaftsverbände. Informations- und Kommunikationsverbände in Deutschland, Großbritannien und Spanien. Wiesbaden: VS Verlag.

Laumann, E.O., Marsden, P.V. y Prensky, D. (1983), “The boundary specification problem in network analysis", en R.S. Burt y M.J. Minor, eds., Applied Network Analysis. Beverly Hills: Sage.

Lowery, D. y Gray, V. (2004), “A Neopluralist Perspective on Research on Organized Interests”, Political Research Quarterly, 57: 163-175.

Masters, R.D. (1990), “Evolutionary Biology and Political Theory”, The American Political Science Review, 84: 195-210.

McFarland, A.S. (2004), Neopluralism: the evolution of political process theory. Lawrence, Kansas: University Press of Kansas.

Molins, J.M. y Casademunt, A. (1999), "Pressure Groups and the Articulation of Interests". en P. Heywood, ed., Politics and Policy in Democratic Spain. Londres: Cass Publishers.

Molins, J.M. y Morata, F. (1993), "Spain: Rapid Arrival of a Latecomer" en M. P. C. M. v. Schendelen, ed., National Public and Private EC Lobbying. Dartmouth Publishing Group: Inglaterra.

Pardo Avellaneda, R. y Fernández Castro, J. (1991), “Las Organizaciones Empresariales y la Configuración del Sistema de Relaciones Industriales de la Espana democrática, 1977-1990", en F. Miguélez y C. Prieto, eds., Las Relaciones Laborales en 62 España. Madrid: Siglo XXI Editores. 
Ronit, K. y Schneider, V. (1997), “Organisierte Interessen in nationalen und supranationalen Politökologien: Ein Vergleich der G7-Länder mit der Europäischen Union”, en U.v. Alemann y B. Wessels, eds., Verbände in vergleichender Perspektive. Berlin: Edition Sigma.

Schmitter, P.C. y Streeck, W. (1999), The Organization of Business Interests. Colonia: MPIfG Discussion Paper.

SEDISI, Asociación Española de Empresas de Tecnología de la Información (2000), Memoria 1999. Madrid: SEDISI.

Steinmo, S. (2003), "The evolution of policy ideas: tax policy in the $20^{\text {th }}$ century", British Journal of Politics and International Relations, 5: 206-236.

Townsend, C.R., Harper, J.L. y Begon, M. (1996), Ecology: Individuals, Populations and Communities. Oxford: Blackwell.

Truman, D.B. (1951), The Governmental Process. Political Interests and Public Opinion. Nueva York: Knopf.

Ward, H. (2003), “The Co-evolution of Regimes of Accumulation and Patterns of Rule: State Autonomy and the Possibility of Functional Responses to Crisis", New Political Economy 8: 179-202.

Wasserman, S. y Faust, K. (1994), Social Network Analysis. Methods and Applications. Cambridge: Cambridge University Press. 